INTERNATIONAL JOURNAL OF RESEARCHES IN BIOSCIENCES, AGRICULTURE AND TECHNOLOGY (c) VISHWASHANTI MULTIPURPOSE SOCIETY (Global Peace Multipumose Socie ty) R. No. MH-659/13(N) www.vmsindia.org

\title{
TAXONOMIC DIVERSITY OF BENTHIC ALGAE FROM JUNNAR TALUKA OF PUNE DISTRICT (INDIA)
}

\author{
Radhakishn Namdeo Tagad \\ Department of Botany, Hon. B. J. College, Ale, Tal. Junnar, Dist. Pune. \\ tagadrn@rediffmail.com
}

\begin{abstract}
:
\section{Introduction}

Filamentous algae were collected from mass growths by hand. Sub-aerial algae growing attached to tree barks, on damp walls or other such substrata were collected by scraping with a scalpel and then picked up with the help of a forceps. Hand collected samples were investigated from April 2014 to October 2015. The present investigation is undertaken with keep in mind that to study the algal population from selected stations of study area.
\end{abstract}

The Junnar tehsil in Pune District is situated between 19011'59" Northern 73052'47" Eastern latitude on the northern part of Deccan Plateau \& composed of undulating hills.Junnar tehsil is famous for its wells and Dams. The famous and historical fort of Shivne ri where ShivajiMaharaj was born is in this region. The re is also a Satellite Center in Arvi. Survey was done of the following impoundments - Manikdoha Dam, Yedgaon Dam, Pimpalgaonjoge Dam, and Wadaj Dam. Sub-aerial algae growing attached to tree barks, on damp walls or other such substrata were collected by scraping with a scalpel and then picked up with the help of a forceps. All algal plants were identifie d up to genus, species, variety as well as formas level. We visit all stations from Junnar Tehsil. All algal plants were identified up to ge nus, species, varie ty as well as formas le vel. We visit all stations from Junnar Tehsil. The 78 species, 6 varieties consisted be longing to 12 familes from 9 orders of 4 classes from 3 divisions. Family Zygnemataceae includes 1 genus, 16 species and 1 variety; while family Batrachospermaceae include only 2 genera and 2 species; Spirogyra is more densely occur in Junnar tehsil.

Keywords: Pune, Junnar, Benthic, Zygnemataceae, Batrachos pe rmaceae, Spirogyra

\section{Materials and Methods}

The samples were preserved in a mixture of $50 \mathrm{ml}$ of $95 \%$ ethyl alcohol, $5 \mathrm{ml}$ of glacial acetic acid, $10 \mathrm{ml}$ of $40 \%$ commercial formalin and 35 $\mathrm{ml}$ of water. The specimens areobserved under microscope for 10X, 40X,100X and Photographs were taken with the help digital camera under appropriate magnifications. Identification ofspecimens was mostly based on the keys give nin standard monographs \& lite ratures. The Vaucher specimens have been deposited at Dept. of Botany, Hon. Baladaheb Jadhav College, Ale, Tal. Junnar, Dist. Pune.

The samples we re bringing to laboratory for identification; Identification were done with the help of Indian monographs and other standard literature like Desikachari (1959), Randhawa (1959), Venkatraman (1961), Prescott (1951), Ramnathan (1964), Bourrlly (1970), Philipose (1967), Gonzalvies (1981), Iyengar and Desikachari (1981), Desikachari et al (1990), Anand (1998) and Sarode and Kamat (1984). The collected algal forms had been preserved in $4 \%$ formalin.

\section{List of Benthic Algal specimens collected from Study Area:}

Division: Chlorophyta
Class: Chlorophyceae
Order: Ulotricales
Suborder: Ulotrich ineae
Family: Ulotrichaceae
1. Ulothrix fimbriata Bold
2. Ulothrix limnetica Lemm.
3. Ulothrix tenerrima (Kutz.)
4. Ulothrix variabilis Kuetzing
5. Uronema africanum Borge
6. Uronema confervicolum Lagerheim

\author{
Order: Chaetophorales \\ Family: Chaetoph ora ceae \\ 1. Stigeoclonium attenuatum (Hazen) \\ Collins \\ 2. Stiegeoclonium nanum Kuetzing \\ 3. Stigeoclonium polymorphum (Franke) \\ Heering \\ 4. Chaetophora elegans (Roth) C. A. \\ Agardh
}


Order: Oed ogoniales

Family: Oedogoniaceae

1. Oedogonium epiphyticum Trans. and Tiff

2. Bulbochaete alabamensis TransandBrown.v. belgaumense Gonz. And Sonn.

3. Bulbochaete kosmoceps Skuja

Order: Cladophorales

Family: Cladophoraceae

1. Pithophora mooreana Collins

2. Pithophora oedogonia (Mont.) Wittrock

3. Rhizoclonium fontanum Kue tzing

Order: Zygnematels

Fa mily: Zygnemataceae

1. Spirogyra a planospora Rand hawa

2. Spirogyra articulate Transeau

3. Spirogyra borgeana Transeau

4. Spirogyra bullata Jao

5. Spirogyra exilisW. and. G.S. west

6. Spirogyra flavescens (Hass) Kutz.

7. Spirogyra irregularies Nageli

8. Spirogyra jogensis Iye ngar v. minor Iyengar

9. Spirogyra karnalae Rand hawa

10. Spirogyra maravillosa Transeau

11. Spirogyra notabilis Taft.

12. Spirogyra paradoxa Rao

13. Spirogyra porangabeae Transeau

14. Spirogyra puncticulata Jao

15. Spirogyra rhizoides Rand hawa

16. Spirogyra submarina (Collins) Transeau

17. Spirogyra sulcata Blum

Class: Ch arophy ceae

Order: Charales

Family:Characeae

1. Chara canescens Desvaux and Loiseieur De slongschamps

2. Chara coralline Wildenow in Mem. AC. Roy.

3. Nitella furcata (Roxb. and Bruzelius) Agardh

4. Nitella hyalina(De Cond.) Agardh.

Division: Cyanophyta

Class: Cyanophyceae

Order: Chroooccales

Family: Chroooccace ae

1. Chroococcus turgidus (Kutz) Naeg.

2. Chroococcus turgidus (Kuetz.) Naeg. v. maximus Nygaard

3. Gloeocapsa calcarea Tilden

4. Gloeocapsa montana Kuetz.
5. Gloeocapsa polydermatica Kue tz

6. Gloeocapsa quaternata (Breb). Kuetz

7. Gloeocapsa stegophila(Itzigs.) Rabenh. v. crassa Rao, C. B.

8. Aphanothece conferta Richter

9. Synechococcus cendrorum Sauvagean

10. Gomphosphaeria aponina Kuetz.

Order: Nostocales

Family: Oscillatoriaceae

1. Oscillatoria simplicissima Gomont

2. Oscillatoria subbrevis Schmidle

3. Oscilatoria terebriformis Ag. Ex Gomont

4. Phormidium corium (Ag.) Gomont

5. Phormidium increstatum (Nageli) Gomont

6. Phormidium laminosum Gomont

7. Phormidium rubroterricola Gardne $\mathrm{r}$

8. Phormidium usterii Schmidle

9. Lyngbya baculum Gomont

10. Lyngbya connectens Bruhl et Biswas

11. Lyngbya digueti Gomont

12. Lyngbya lachneri (Zimmermann) Geitler

13. Schizothrix arenaaria (Berk) Gomont v. non-constricta Gose

14. Schizothrix ericetorum Lemmermann

15. Microcoleus acu tissimus Ge rdene $\mathrm{r}$

16. Microcoleus chthonoplastes Thure tex Gomont

Family: Nostocaceae

1. Anabaena iyengarii Bharadwaja v. tenu is Rao, C. B.

2. Nostoc calcicola Brebis son ex Born. etFlah.

3. Nostoc entophytum Born. etFlah.

4. Nosto chumifu sum Carmichael ex Born. etFlah.

5. Nostoc paludosum Kutzing ex Born. Et Flah.

6. Nostoc punctiforme (Kue tz.) Hariot

7. Nostoc spongiaeformi Agardh ex. Born. etFlah.

8. Au losira bombayensis Gozalves

Family: Scytonemataceae

1. Scytonema chiastum Geitler

2. Scytonema pseudohofmanni Bharadwaja

3. Scytonema schmidlei J. De Toni

4. Tolypothrix byssoidea (Berk.) Kirchner

5. Tolypothrix distorta Kuetzing ex Born.etFlah.

Family: Rivulariaceae 
1. Calothrix atrichaFremy

2. Calothrix clavata West, G. S.

3. Calothrix elenkinii Kossinskaja

4. Calothrix thermalis (Schwabe) Hansg

5. Rivularia beccariana (De Not.) Born.et Flah.

6. Rivularia manginii Frem

\section{Division: Rhodophyta}

Class: Florideophyceae

Order: Batrachospermales

Family: Batrach ospermaceae

1. Batrachospermum mahabaleshwarensis Balk. et Chaug.

2. Sirodotia huiellense (Welw.) Skuja

\section{References}

1. Anand, N.; Handbook of Blue-Green Algae (of Rice Fields of South India)'; Publ. Bishen Singh Mahendra Pal Singh, Dehradun:1989

2. Anand, N.; 'Indian Fresh Water Microalgae'; Published by BishenSingh. Mahendra.

3. Anand, N. and G. Revathi; 'Blue-Green Algae from Rice Fields of Tamil Nadu'; Phykos 26:17$21: 1987$
4. Bhoge, O. N. and G. Ragothaman; 'Studies on the Cyanophyceae from Jalgaon Region, Maharashtra'; Phykos 25:129-131:1986

5. Bhoge, O. N. and G. Ragothaman; 'Studies on the Euglenophyceae from Jalgaon Region, Maharashtra'; Phykos 25:132-135:1986

6. Desikachary, T. V.; 'Cyanophyta'; Indian Council of Agricultural Research, New Delhi: 1686:1959

7. Ivengar, M. O. P. and T. V. Desikacharv; 'Volvocales'; Indian Council of Agricultural Research, New Delhi:1-532:1981

8. Lackey, J. B.; 'The manipulation and counting of river plankton and changes in some organisms due to formalin preservation'; U. S. Public Health Reports:53:2080-2093:1938

9. Nandan, S. N. and N. H. Aher; 'Species diversity in algal flora of Haranbaree dam and Mosam river of Baglan (Maharashtra). J. Ecol. Envi. and Cons., 81(3-4): 551-553:2005

10. Pingle, S. D.; 'Studies on the algae of impoundments and streams in Maharash tra'; $\mathrm{Ph}$. D. thesis, Poona University:1981

11. Presscott, G. W.; 'How To Know the FreshWater Algae'; W. M. C Brown Company Publishers, Dubuque, IOWA:1954 